\title{
Short-term Outcomes of Transanal versus Laparoscopic Total Mesorectal Excision: A Systematic Review and Meta-Analysis of Cohort Studies
}

\author{
Zhiyuan $\mathrm{Wu}^{1^{*}}$, Wenlong Zhou ${ }^{2}$, Fu Chen ${ }^{3}$, Wentao Wang ${ }^{4}$, Yong Feng ${ }^{1^{凶}}$ \\ 1. Department of Colorectal and Hernia Minimally Invasive Surgery, Shengjing Hospital of China Medical University, Shenyang, China \\ 2. Department of General Surgery, Shenyang 242 Hospital, Shenyang, China \\ 3. Department of General Surgery, The Fourth Affiliated Hospital of China Medical University, Shenyang, China \\ 4. Department of Gastrosurgery, Liaoning Cancer Hospital and Institute, Shenyang, China \\ *Zhiyuan $\mathrm{Wu}$ and Wenlong Zhou contributed equally to this work.
}

$\square$ Corresponding author: Yong Feng, PhD., Department of Colorectal and Hernia Minimally Invasive Surgery, Shengjing Hospital of China Medical University, No.36 Sanhao Street, 110000, Shenyang, China. Email: fengy@sj-hospital.org

(c) Ivyspring International Publisher. This is an open access article distributed under the terms of the Creative Commons Attribution (CC BY-NC) license (https://creativecommons.org/licenses/by-nc/4.0/). See http://ivyspring.com/terms for full terms and conditions.

Received: 2018.06.12; Accepted: 2018.10.01; Published: 2019.01.01

\begin{abstract}
Introduction: The TaTME surgery has been developed to overcome the difficulties encountered in the practice of laparoscopic surgery for rectal cancer, especially in male, obese patients with a narrow pelvis and mid and low rectal tumours. Although the TaTME shows some promising results regarding oncological and operative outcomes, some pitfalls have been indicated. Thus, the real benefits of this novel technique over the laparoscopic surgery remain unknown. The aim of the present study was to perform a systematic review and meta-analysis of the currently available literature on the outcomes of TaTME in comparison with laparoscopic procedure.

Methods: A systematic literature search was conducted using the web-based databases MEDLINE, EMBASE, and Cochrane CENTRAL, followed by a manual search of the references of the initially identified articles. The study information, patient characteristics, oncological outcomes, perioperative outcomes, as well as short- and long-term postoperative outcomes were collected and critically evaluated.

Results: Nine retrospective cohort studies were identified, comprising 751 patients (348 with TaTME, 403 with LaTME). The positive circumferential resection margin $(P=0.01)$ was better in patients treated with TaTME; whereas the quality of mesorectum, circumferential resection margin, distal resection margin, and harvested lymph nodes were comparable. The TaTME was associated with shorter operative time $(P=0.05 ; P=0.0006$ in subgroup analysis), less blood loss $(P=0.02)$, less conversion $(P=0.007)$, and shorter hospital stay $(P=0.06 ; P=0.003$ in subgroup analysis). The intraoperative complications were similar. As for the postoperative outcomes, the overall postoperative complications $(P=0.02)$ and the readmission $(P=0.003)$ were found less in patients treated with TaTME; however, the individual postoperative complications were found comparable.

Conclusion: The present systematic review and meta-analysis suggested some advantages of TaTME, in terms of circumferential resection margin involvement, operative time, blood loss, conversion, hospital stay, overall postoperative complications, and readmission. It appears that the TaTME procedure achieved a better resection quality and smoother recovery in selected patients, without compromising the short-term safety. Nevertheless, it is too early to draw any conclusion, since results of high quality clinical evidence from randomized controlled trials have to be awaited. As it is technically demanding, the procedure needs to be taught and learned systematically to ensure safe implementation.
\end{abstract}

Key words: rectal cancer, transanal total mesorectal excision, laparoscopic, comparison, meta-analysis, systematic review 


\section{Introduction}

Colorectal cancer is the third most common cancer in men and the second in women worldwide [1]. Total mesorectal excision (TME), since introduced by Heald and colleagues in the 1980s, has become the standard of care for treatment of rectal cancer, as it confers improvements in recurrence and survival [2]. Open surgery used to be the only option available; however, laparoscopic procedure has been widely accepted by surgeons, since it shows in some randomized trials a certain degree of benefits with respect to postoperative recovery [3-6]. Nevertheless, the conversion rate to an open approach is high, with more complications and worse outcomes [7]. The main reasons for conversion are difficulties in pelvic exposure, which is especially frequently needed in male, obese patients with a narrow pelvic cavity and low rectal tumour.

To overcome these issues, a bottom-up procedure of transanal TME surgery has been introduced firstly by Whiteford and colleagues on cadavers [8] and then by Sylla and colleagues on live patients [9]; and since then, it has gained substantial popularity [10]. Theoretically, approaching the pelvic mesorectum transanally has the advantages of more precise dissection under adequate visualization, which may potentially lead to better specimen quality, circumferential and distal resection margins. Case series studies show that transanal TME is oncologically safe and effective in highly selected patients, with acceptable short-term outcomes and good specimen quality $[11,12]$. Despite the potential benefits, concern exists for the potential risks of tumour cell dissemination and bacterial contamination [13, 14]. Besides, new serious complications have been documented $[5,6,11]$. Thus, whether the transanal TME is indeed superior to laparoscopic surgery is controversial, and is in urgent need to be clarified.

Therefore, the aim of the present study was to perform a systematic review and meta-analysis of the currently available literature on the outcomes following transanal total mesorectal excision (TaTME) in comparison with laparoscopic TME (LaTME), critically evaluating the short-term results, with specific focus on the oncological outcomes.

\section{Methods}

This systematic review and meta-analysis was performed according to the Preferred Reporting Items for Systematic Reviews and Meta-Analysis (PRISMA) guidelines (available at http://www.prisma-stateme nt.org/).[15] A two-level search strategy was applied to identify all available studies in the English literature investigating oncological, perioperative, and postoperative outcomes of TaTME. First, a search of databases including MEDLINE, EMBASE and Cochrane CENTRAL was performed through December 2017 using the web-based search engines PubMed and Ovid. Second, the references of the initially identified articles were manually reviewed for any additional studies. Search terms included the following words with different combinations: transanal total mesorectal excision, TaTME, transanal minimally invasive surgery, TAMIS, natural orifice transluminal endoscopic surgery, NOTES; laparoscopy, laparoscopic; rectal cancer, rectal carcinoma, rectal adenocarcinoma.

Published studies were considered for inclusion if they met the following criteria: the study design was randomized controlled trials, cohort studies, or matched case-control studies; the study population comprised patients definitely diagnosed with rectal cancer, and treated with radical surgery; the surgical procedure was clearly documented as indeed a TaTME; outcomes of interest were compared between the TaTME and LaTME groups; the sample size was $\geq$ 20 in each group. Whereas, studies on animals or cadavers; studies without comparison with LaTME; studies with benign lesions only, or no distinct group of malignant rectal tumour; studies on transanal endoscopic microsurgery or other local excision; absence of outcomes of interest reported, or impossible to determine from the data acquired; studies in languages other than English; and correspondences, case reports, reviews, meta-analysis, and conference abstracts were excluded. The initial studies were retrieved, and data were extracted independently by two investigators ( $\mathrm{Wu}$ and Zhou), and any disagreements were resolved by consulting another author (Feng). The Newcastle-Ottawa Quality Assessment Scale was used to evaluate the quality of the studies included, where studies with a score 6 or above were considered high quality [16].

The primary endpoints were pathological outcomes of the specimen, since the long-term outcomes were scarce; whereas the perioperative and postoperative outcomes were set as secondary endpoints. The following end-points were collected from the included studies: (a) study information, including first author, year of publication, study period, country/region, study design, number of patients, inclusion and exclusion criteria; (b) patient characteristics, including age, gender, body mass index (BMI), American Society of Anaesthesiology (ASA) score, neoadjuvant therapy, tumour size, tumour location, clinical and pathological TNM staging, history of abdominal surgery, and duration of follow-up; (c) oncological outcomes, including quality of mesorectum, circumferential resection margin 
(CRM), positive CRM, distal resection margin (DRM), positive DRM, and harvested lymph nodes; (d) perioperative outcomes, including type of procedure, anastomotic type, operative time, blood loss, conversion, diverting ostomy creation, splenic flexure mobilization, intraoperative complications, and hospital stay; (e) short-term postoperative outcomes, including overall and individual postoperative complications, and readmission; (f) long-term outcomes, including overall, local, and distant recurrence, and overall and disease-free survival.

For dichotomous data, we generated risk ratios (RRs) and 95\% confidence intervals (CIs) for comparison between TaTME and LaTME. The

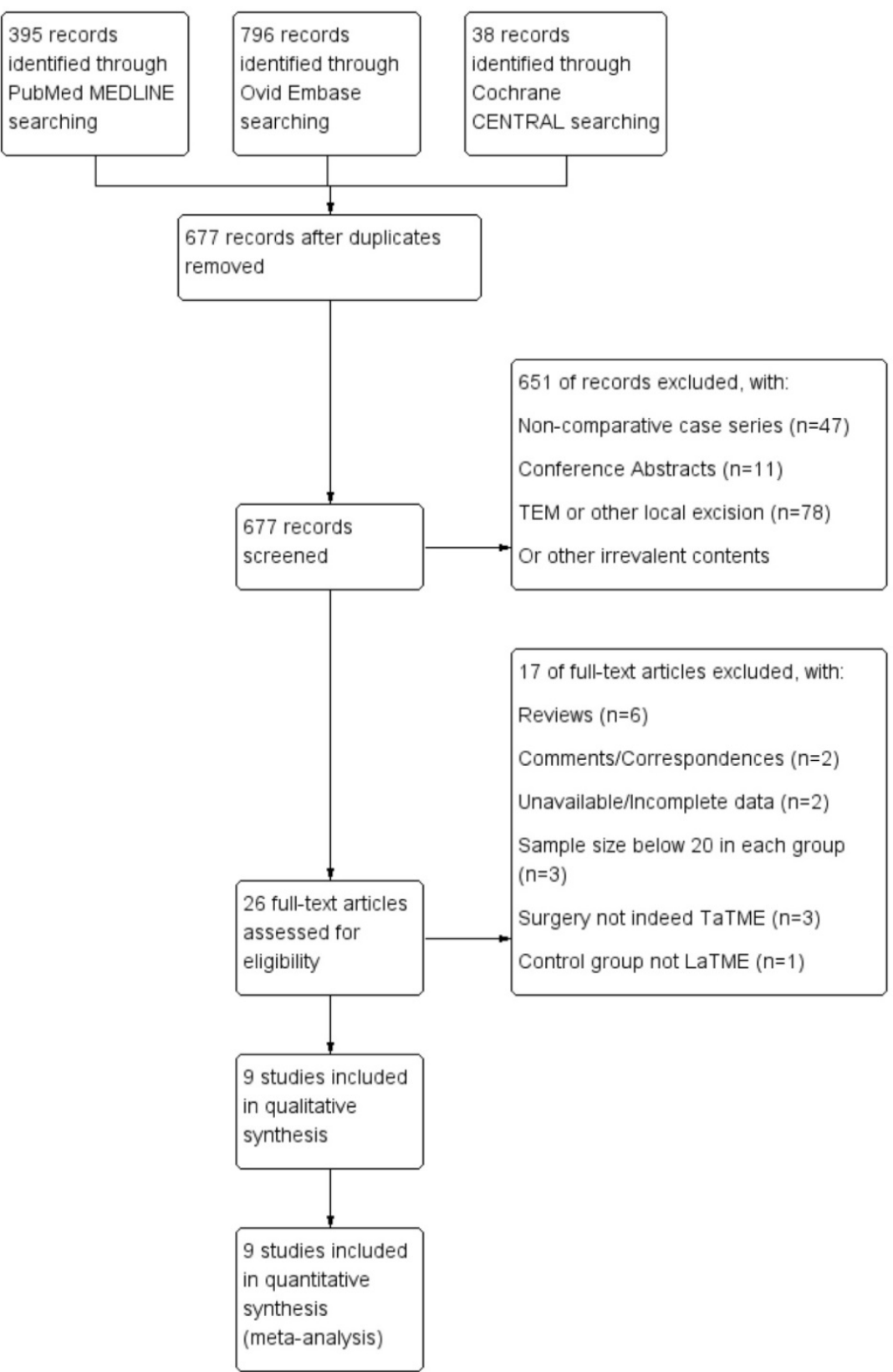

Mantel-Haenszel method was used to combine the RRs for the outcomes of interest. For continuous data, mean differences (MDs) and 95\% CIs were generated. The inverse variance method was used to combine the MDs. If the mean and standard deviation (S.D.) were not provided, they were calculated using the method described by Hozo and colleagues [17]. Concerning the inborn heterogeneity of the non-randomized retrospective studies included, the random-effects (RE) model of estimate was applied as the summary measure; when there was no obvious inter-study heterogeneity identified from an $\mathrm{I}^{2}$ test, the results of the fixed-effect (FE) model were also provided for reference. An $\mathrm{I}^{2}$ value between 0 and $40 \%$ was considered no obvious heterogeneity according to the Cochrane Handbook. [18] Finally, publication bias was evaluated using funnel plots of all studies included in this meta-analysis. Differences were considered statistically significant at $P<0.05$. All analyses were performed using Review Manager (version 5.3, available at http://community.cochrane.org/tools / review-production-tools/revman-5).

\section{Results}

Of the 677 potentially relevant studies initially retrieved, 9 eligible studies [19-27] were eventually identified to meet the inclusion criteria of the present meta-analysis, comprising a total of 751 patients with rectal cancer. A summary of the study selection and inclusion process is illustrated in Figure 1. The articles were published between 2014 and 2017, from Taiwan, France, Denmark, Spain, Russia, and the Netherlands; with patient enrolments from 2007 to 2017. There were 2 articles $[24,25]$ published by the same authors; however, both were included because it clearly stated that the cohorts did not overlap. All of the 9 studies were retrospective cohort studies, and all but one [20] were based on single medical centre. A summary of the general information of the included studies is presented in Table 1. All of the studies gained a score 6 or above on the Newcastle-Ottawa Scale, as is shown in Table 2. 
Table 1. A summary of the included studies.

\begin{tabular}{|c|c|c|c|c|c|c|c|c|}
\hline $\begin{array}{l}\text { First } \\
\text { Author }\end{array}$ & $\begin{array}{l}\text { Year of } \\
\text { Publication }\end{array}$ & Study Period & $\begin{array}{l}\text { Country/ } \\
\text { Region }\end{array}$ & Study Design & $\begin{array}{l}\text { Number of } \\
\text { Patients }\end{array}$ & Matched for & Inclusion Criteria & Exclusion Criteria \\
\hline Chang [19] & 2017 & $\begin{array}{l}\text { TaTME Jan } 2014- \\
\text { May } 2017 \\
\text { LaTME } 2008- \\
2016\end{array}$ & Taiwan & $\begin{array}{l}\text { Retrospective } \\
\text { Cohort Study } \\
\text { Single Centre }\end{array}$ & 46 & $\begin{array}{l}\text { Gender, Age, } \\
\text { ASA Score, } \\
\text { Clinical Stage }\end{array}$ & $\begin{array}{l}\text { Confirmed rectal } \\
\text { adenocarcinoma; Received } \\
\text { TaTME or LaTME; }\end{array}$ & $\begin{array}{l}\text { Location }>7 \mathrm{~cm} \text { from anal } \\
\text { verge; } \\
\text { Preoperative imaging locally } \\
\text { advanced tumour (T4); } \\
\text { Stage IV; }\end{array}$ \\
\hline Chen [20] & 2015 & $\begin{array}{l}\text { TaTME May } 2013 \\
\text { - Apr } 2015 \\
\text { LaTME } 2007 \text { - } \\
2014\end{array}$ & Taiwan & $\begin{array}{l}\text { Retrospective } \\
\text { Cohort Study } \\
\text { Multiple } \\
\text { Centres }\end{array}$ & 150 & $\begin{array}{l}\text { Gender, Age, } \\
\text { ASA Score, } \\
\text { Clinical Stage }\end{array}$ & $\begin{array}{l}\text { Biopsy-proven middle or low } \\
\text { rectal adenocarcinoma; } \\
\text { Patients received nCRT; } \\
\text { Stage II/III; }\end{array}$ & $\begin{array}{l}\text { Initial } 20 \text { cases during learning } \\
\text { curve; } \\
\text { Patients did not receive nCRT; } \\
\text { Stage IV; }\end{array}$ \\
\hline $\begin{array}{l}\mathrm{de}^{\prime} \text { Angelis } \\
\text { [21] }\end{array}$ & 2015 & $\begin{array}{l}\text { TaTME Jan } 2011 \text { - } \\
\text { Dec } 2014 \\
\text { LaTME Jan } 2008 \text { - } \\
\text { Dec } 2010\end{array}$ & France & $\begin{array}{l}\text { Retrospective } \\
\text { Cohort Study } \\
\text { Single Centre }\end{array}$ & 64 & $\begin{array}{l}\text { Gender, Age, } \\
\text { BMI, Type of } \\
\text { Procedure }\end{array}$ & $\begin{array}{l}\text { Histologically proven low } \\
\text { rectal adenocarcinoma; } \\
\text { Location } \leq 5 \mathrm{~cm} \text { from anal } \\
\text { verge; }\end{array}$ & $\mathrm{N} / \mathrm{A}$ \\
\hline $\begin{array}{l}\text { Fernandez- } \\
\text { Hevia }{ }^{[22]}\end{array}$ & 2014 & $\begin{array}{l}\text { TaTME Nov } 2011 \\
\text { - Mar } 2013 \\
\text { LaTME Aug } 2010 \\
\text { - Oct } 2011\end{array}$ & Spain & $\begin{array}{l}\text { Retrospective } \\
\text { Cohort Study } \\
\text { Single Centre }\end{array}$ & 74 & $\mathrm{~N} / \mathrm{A}$ & $\begin{array}{l}\text { Histologically confirmed } \\
\text { middle or low rectal } \\
\text { adenocarcinoma; } \\
\text { Location } \leq 10 \mathrm{~cm} \text { from anal } \\
\text { verge; }\end{array}$ & $\begin{array}{l}\text { High rectal cancer; } \\
\text { Abdominoperineal resection; }\end{array}$ \\
\hline Lelong [23] & 2017 & $\begin{array}{l}\text { TaTME Jun } 2011 \text { - } \\
\text { Dec } 2013 \\
\text { LaTME Jan } 2008 \text { - } \\
\text { Jun } 2011\end{array}$ & France & $\begin{array}{l}\text { Retrospective } \\
\text { Cohort Study } \\
\text { Single Centre }\end{array}$ & 72 & Not Matched & $\begin{array}{l}\text { Resectable low rectal cancer; } \\
\text { Coloanal anastomosis; }\end{array}$ & $\begin{array}{l}\text { T4 tumours requiring } \\
\text { extended resection; } \\
\text { nonresectable metastases; } \\
\text { evidence of peritoneal } \\
\text { carcinosis; }\end{array}$ \\
\hline $\begin{array}{l}\text { Perdawood } \\
\text { [24] }\end{array}$ & 2015 & $\begin{array}{l}\text { TaTME Dec } 2013 \text { - } \\
\text { Apr } 2015 \\
\text { LaTME Feb } 2013 \text { - } \\
\text { Nov } 2013\end{array}$ & Denmark & $\begin{array}{l}\text { Retrospective } \\
\text { Cohort Study } \\
\text { Single Centre }\end{array}$ & 50 & Gender & $\begin{array}{l}\text { Rectal adenocarcinoma located } \\
\text { within } 10 \mathrm{~cm} \text { from anal verge; } \\
\text { Patients underwent } \\
\text { intersphincteric } \\
\text { abdominoperineal excision; } \\
\text { T4 tumours included if radical } \\
\text { resection achievable after } \\
\text { nCRT; }\end{array}$ & $\begin{array}{l}\text { Patients underwent standard } \\
\text { or extralevator } \\
\text { abdominoperineal excision; }\end{array}$ \\
\hline $\begin{array}{l}\text { Perdawood } \\
{[25]}\end{array}$ & 2017 & $\begin{array}{l}\text { TaTME May } 2015 \\
\text { - Mar } 2017 \\
\text { LaTME N/A }\end{array}$ & Denmark & $\begin{array}{l}\text { Retrospective } \\
\text { Cohort Study } \\
\text { Single Centre }\end{array}$ & 200 & $\begin{array}{l}\text { Propensity } \\
\text { Score Matching } \\
\text { for Gender, } \\
\text { BMI, Tumour } \\
\text { Status, Tumour } \\
\text { Height }\end{array}$ & $\begin{array}{l}\text { Mid and low rectal cancer; } \\
\text { TME as operative principle; } \\
\text { Location } 4-11 \mathrm{~cm} \text { from anal } \\
\text { verge; } \\
\text { T4 tumours included if radical } \\
\text { surgery achievable after nCRT; }\end{array}$ & $\begin{array}{l}\text { Extralevator or standard } \\
\text { abdominoperineal excision; } \\
\text { Patients reported in the } \\
\text { authors' previous publication; }\end{array}$ \\
\hline Rasulov [26] & 2016 & Oct 2013 - Jan 2015 & Russia & $\begin{array}{l}\text { Retrospective } \\
\text { Cohort Study } \\
\text { Single Centre }\end{array}$ & 45 & Not Matched & $\begin{array}{l}\text { Biopsy-proven } \\
\text { mrT1-4aN0-2M0 rectal cancer } \\
\text { located in low rectum, } \leq 10 \mathrm{~cm} \\
\text { from anal verge; }\end{array}$ & $\begin{array}{l}\text { Eastern Cooperative Oncology } \\
\text { Group performance status } \geq 2 ; \\
\text { Patients undergoing partial } \\
\text { mesorectal excision; }\end{array}$ \\
\hline Velthuis [27] & 2014 & $\begin{array}{l}\text { TaTME Jun } 2012 \text { - } \\
\text { Jul } 2013 \\
\text { LaTME Jun } 2011 \text { - } \\
\text { Jun } 2012\end{array}$ & $\begin{array}{l}\text { Nether- } \\
\text { lands }\end{array}$ & $\begin{array}{l}\text { Retrospective } \\
\text { Cohort Study } \\
\text { Single Centre }\end{array}$ & 50 & $\begin{array}{l}\text { Gender, Type } \\
\text { of Procedure }\end{array}$ & $\begin{array}{l}\text { Histology proven distal or mid } \\
\text { rectal carcinoma; }\end{array}$ & $\begin{array}{l}\text { Distant metastasis; } \\
\text { T4 tumours; } \\
\text { Previous abdominal surgery; }\end{array}$ \\
\hline
\end{tabular}

TaTME : transanal total mesorectal excision; LaTME : laparoscopic total mesorectal excision; N/A : not available; ASA : American Society of Anaesthesiology; BMI : body mass index; nCRT : neoadjuvant chemoradiotherapy; TME : total mesorectal excision.

Table 2. Newcastle-Ottawa Scale of the included studies.

\begin{tabular}{|c|c|c|c|c|c|c|c|c|c|}
\hline \multirow[t]{2}{*}{ Study } & \multicolumn{4}{|l|}{ Selection } & \multirow[t]{2}{*}{ Comparability } & \multicolumn{3}{|l|}{ Outcome } & \multirow{2}{*}{$\begin{array}{r}\text { Total } \\
- \text { Score }\end{array}$} \\
\hline & $\begin{array}{l}\text { Representativeness } \\
\text { of exposed cohort }\end{array}$ & $\begin{array}{l}\text { Selection of the } \\
\text { non-exposed } \\
\text { cohort }\end{array}$ & $\begin{array}{l}\text { Ascertainment } \\
\text { of exposure }\end{array}$ & $\begin{array}{l}\text { Outcome of } \\
\text { interest not } \\
\text { present at start }\end{array}$ & & $\begin{array}{l}\text { Assessment } \\
\text { of outcome }\end{array}$ & $\begin{array}{l}\text { Length of } \\
\text { follow-up }\end{array}$ & $\begin{array}{l}\text { Adequacy of } \\
\text { follow-up }\end{array}$ & \\
\hline Chang 2017 [19] & * & * & * & * & ** & * & & & 7 \\
\hline Chen 2015 [20] & * & * & * & * & $* *$ & * & & & 7 \\
\hline de' Angelis 2015 [21] & * & * & * & * & $* *$ & * & * & & 8 \\
\hline $\begin{array}{l}\text { Fernandez-Hevia } \\
2014[22]\end{array}$ & * & * & * & * & * & * & & & 6 \\
\hline Lelong 2017 [23] & * & * & * & * & * & * & * & & 7 \\
\hline Perdawood 2015 [24] & * & * & * & * & * & * & & & 6 \\
\hline Perdawood 2017 [25] & * & * & * & * & $* *$ & * & & & 7 \\
\hline Rasulov $2016^{[26]}$ & * & * & * & * & * & * & & & 6 \\
\hline Velthuis 2014 [27] & * & * & * & * & ** & * & & & 7 \\
\hline
\end{tabular}

Each asterisk $\left(^{*}\right)$ represents one score.

The patient characteristics are presented in Table 3. There were 348 patients treated with TaTME, and 403 with LaTME. All studies reported data on gender and BMI, while age was reported in 8 studies, and ASA score in 7 , tumour size in 6 . None of them showed any difference, though the cohorts of 3 were not matched [22, 23, 26]. All reported information on neoadjuvant therapy, and 2 of them showed more patients in TaTME group received neoadjuvant therapy $[22,26]$. The distance of the tumour from anal verge was reported in 8 studies, and only in the study of Chen and colleagues [20] were the tumours 
significantly closer in the TaTME group to the anal verge. However, when they divided the patients into middle $(>7 \mathrm{~cm})$ and low $(\leq 7 \mathrm{~cm})$ rectal cancers, they found no difference regarding the patient distribution. All studies reported the clinical TNM staging, and none of them indicated any difference between the groups, except for one by Perdawood and colleagues [25], where the regional lymph node classification was better in the TaTME group; whereas, according to the authors, it exerted no influence on the choice of the operation or preoperative chemoradiation strategies following the clinical guidelines in their country. Regarding the pathological TNM staging, 8 studies showed comparable results between the groups. Besides, 2 studies [21, 27] reported comparable results with respect to tumour differentiation. All patients included underwent laparoscopic TME or laparoscopic-assisted transanal TME, and 7 studies [19, 21, 23-27] documented the exact surgical procedures performed, including low anterior resection, partial and total intersphincteric resection, abdominoperineal resection, and Hartmann procedure, where none of them indicated any difference between the groups. Additionally, the abdominal surgery history was reported in 6 studies, including one excluded any patients with previous abdominal surgery in their study design [27]. Moreover, the duration of follow-up was reported in 2 studies, and one indicated significantly shorter follow-up in the TaTME group [23].

The oncological outcomes are illustrated in Figure 2, Supplementary Figures S1, S2, S3, and Table 4. The specimen quality was graded as complete, nearly complete, and incomplete as suggested by Quirke and colleagues [28, 29]. Seven studies [21-27] investigated the quality of mesorectum, where 2 of them $[25,27]$ showed significant differences between the groups. In the pooled analyses, no difference was found with respect to complete (RR 1.03, 95\% CI $0.92-1.16, P=0.57$ ), nearly complete (RR $1.35,95 \% \mathrm{CI}$ 0.95-1.91, $P=0.10$ ), or incomplete mesorectum (RR $0.64,95 \%$ CI 0.39-1.06, $P=0.08$ ). Two articles [21, 27] indicated similar length of the specimen. Six studies[20-22, 24, 25, 27] reported data on CRM, and none of them showed any difference; and in the pooled analysis we did not observe any difference between the two groups (MD 0.64, 95\% CI -0.09-1.37, $P$ $=0.08$ ). All of the 9 studies investigated positive CRM, where a less positive CRM was found in the TaTME group (RR 0.47, 95\%CI 0.26-0.84, $P=0.01$ ). The definitions of a positive CRM was heterogeneous among the studies, where 2 of them [21, 27] defined positive as malignant cells found within $2 \mathrm{~mm}$ from the CRM, and all others took it $1 \mathrm{~mm}$. When we included only the studies defining positive as $1 \mathrm{~mm}$, the difference remained (RR 0.48, 95\% CI 0.26-0.89, $P=0.02$ ). Seven studies [19-22, 24, 25, 27] reported data on DRM and 4 $[19,21,23,25]$ on positive DRM; however, none of the pooled analyses found any difference between the groups (DRM, MD 0.34, 95\%CI -0.09-0.78, $P=0.12$; Positive DRM, RR 0.71, 95\%CI 0.15-3.40, $P=0.67$ ). Finally, all studies reported the number of harvested lymph nodes, and it was found comparable between the groups (MD 0.21, 95\% CI -0.96-1.38, $P=0.72$ ).

The perioperative outcomes are demonstrated in Figure 3, Supplementary Figures S4, S5, and Table 4. Eight studies [19-26] investigated the operative time, and 4 of them [21, 22, 24, 25] indicated a significantly shorter operative time associated with TaTME. In the pooled analysis, we observed a seemingly shorter operative time in the TaTME group, but it did not reach significance $(P=0.05)$. The number of surgical teams performing TaTME was different among studies, comprising one team working sequentially $[19,21,23-25]$, two teams working simultaneously [22], and a hybrid of them for different patients[20, 26]. When including only one-team TaTME studies, however, a significantly shorter operative time was observed in the TaTME group (MD -32.06, 95\%CI $-50.33--13.80, P=0.0006)$. The intraoperative blood loss was investigated in 5 studies [19, 20, 24-26], and significantly less blood loss was observed in the TaTME group (MD -66.78, 95\%CI -124.18 - -9.38, $P=$ 0.02). Conversion [19-26] was found significantly fewer in the TaTME group (RR 0.25, 95\% CI 0.09-0.68, $P=0.007)$. Splenic flexure mobilization [20, 22, 24, 25] was more in the TaTME group (RR 1.64, 95\%CI 1.19-2.27, $P=0.003)$, but the diverting ostomy creation $[19-23,26]$ was comparable. The intraoperative complications was investigated in 5 studies [20-22, 24, 25], and no difference was observed between the groups. Concerning the individual intraoperative complications, bleeding and adjacent organ injury were also found comparable between the groups. Eight studies investigated the hospital stay [19-26], and 4 of them demonstrated significant differences [21, 23-25]. Nevertheless, in the pooled analysis, we failed to observe any significant difference between the groups, though patients undergoing TaTME tended to have a shorter hospital stay $(P=0.06)$. The postoperative recovery strategies were heterogeneous, with some implemented an Enhanced Recovery after Surgery (ERAS) protocol [20]. When including studies clearly stated not using ERAS [22, 24, 25], a significant shorter hospital stay was observed in patients undergoing TaTME (MD -4.23, 95\%CI -7.01 $-1.45, P=0.003$ ). 


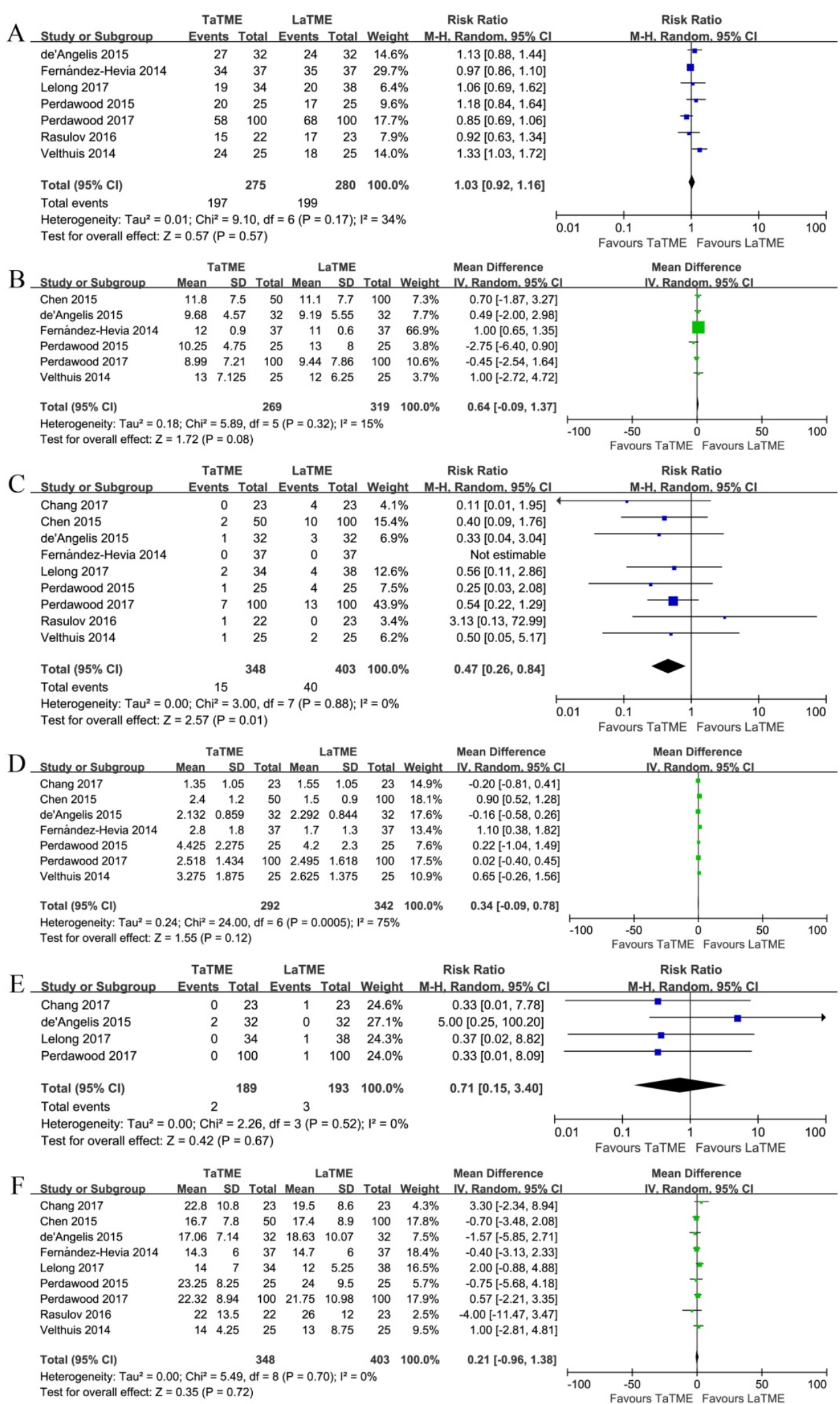

Figure 2. Forest plots of risk ratios and mean differences of oncological outcomes of the included studies in random-effects model, (A) Quirke quality of mesorectum to be complete, $(B)$ circumferential resection margin, $(C)$ positive circumferential resection margin, $(D)$ distal resection margin, $(E)$ positive distal resection margin, and $(F)$ harvested lymph nodes. TaTME : transanal total mesorectal excision; LaTME : laparoscopic total mesorectal excision; SD : standard deviation; M-H : Mantel-Haenszel; IV : inverse variance; $\mathrm{Cl}$ : confidence interval. 


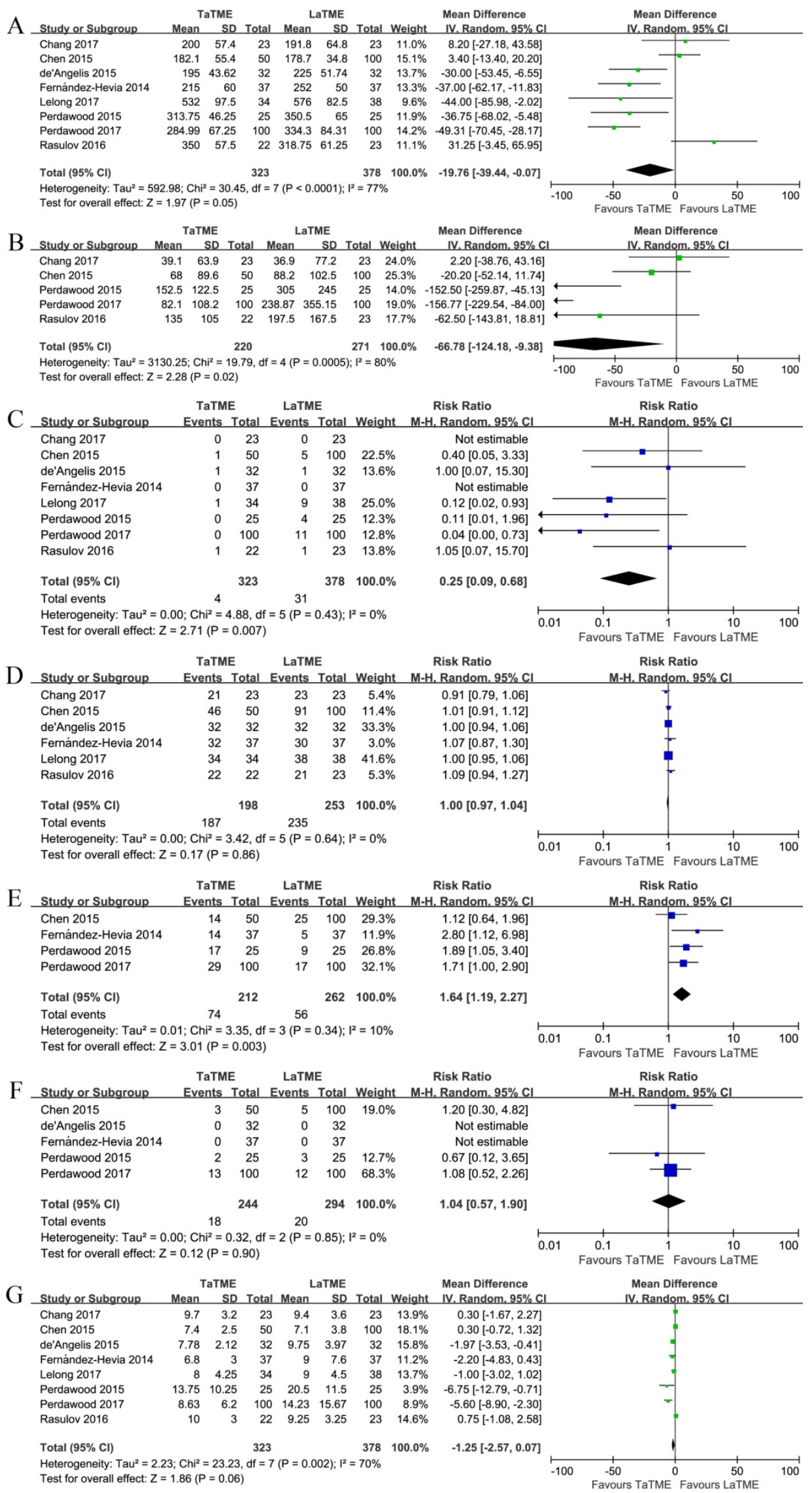

Figure 3. Forest plots of risk ratios and mean differences of perioperative outcomes of the included studies in random-effects model, (A) operative time, (B) blood loss, (C) conversion, (D) diverting ostomy, (E) splenic flexure mobilization, (F) overall intraoperative complications, and (G) hospital stay. TaTME : transanal total mesorectal excision; LaTME : laparoscopic total mesorectal excision; SD : standard deviation; $\mathrm{M}-\mathrm{H}$ : Mantel-Haenszel; IV : inverse variance; Cl : confidence interval. 
Table 3. Patient characteristics of the included studies.

\begin{tabular}{|c|c|c|c|c|c|c|c|c|c|c|c|c|c|c|c|c|c|c|c|c|c|c|c|c|}
\hline \multirow[t]{2}{*}{ Study } & \multicolumn{2}{|c|}{$\begin{array}{l}\text { Number } \\
\text { of } \\
\text { Patients }\end{array}$} & \multicolumn{2}{|c|}{$\begin{array}{l}\text { Age } \\
\text { [Years, } \\
\text { Mean } \pm \text { S.D./ } \\
\text { Median } \\
\text { (Range)] }\end{array}$} & \multicolumn{2}{|c|}{$\begin{array}{l}\text { Gender } \\
\text { (n, Male/ } \\
\text { Female) }\end{array}$} & \multicolumn{2}{|c|}{$\begin{array}{l}\text { BMI } \\
{\left[\mathrm{kg} / \mathrm{m}^{2},\right.} \\
\text { Mean } \pm \text { S.D. } \\
\text { /Median } \\
\text { (Range)] }\end{array}$} & \multicolumn{2}{|c|}{$\begin{array}{l}\text { ASA Score } \\
\text { (n) }\end{array}$} & \multicolumn{2}{|c|}{$\begin{array}{l}\text { Neoadju } \\
\text { vant } \\
\text { Therapy } \\
\text { (n) }\end{array}$} & \multicolumn{2}{|c|}{$\begin{array}{l}\text { Tumour } \\
\text { Size } \\
{[\mathrm{mm},} \\
\text { Mean } \\
\pm \text { S.D./ } \\
\text { Median } \\
\text { (Range)] }\end{array}$} & \multicolumn{2}{|c|}{$\begin{array}{l}\text { Tumour Location } \\
{[\mathrm{cm} \text { from anal }} \\
\text { verge, Mean } \pm \text { S.D./ } \\
\text { Median (Range)] }\end{array}$} & \multicolumn{2}{|c|}{$\begin{array}{l}\text { Clinical Staging } \\
\text { (n) }\end{array}$} & \multicolumn{2}{|c|}{$\begin{array}{l}\text { Pathological Staging } \\
\text { (n) }\end{array}$} & \multicolumn{2}{|c|}{$\begin{array}{l}\text { History of } \\
\text { Abdominal } \\
\text { Surgery } \\
\text { (n) }\end{array}$} & \multicolumn{2}{|c|}{$\begin{array}{l}\text { Mean } \\
\text { Follow-up } \\
\text { [months, } \\
\text { Mean } \\
\pm \text { S.D./ } \\
\text { Median } \\
\text { (Range)] }\end{array}$} \\
\hline & Ta & La & Ta & La & Ta & La & Ta & La & Ta & La & Ta & La & Ta & La & Ta & $\mathbf{L a}$ & Ta & La & Ta & La & Ta & La & Ta & La \\
\hline $\begin{array}{l}\text { Chang } \\
2017 \\
\text { [19] }\end{array}$ & 23 & 23 & $\begin{array}{l}62.4 \pm \\
12.9\end{array}$ & $\begin{array}{l}62.9 \pm \\
12.6\end{array}$ & $\begin{array}{l}13 / \\
10\end{array}$ & $\begin{array}{l}13 / \\
10\end{array}$ & $\begin{array}{l}25.8 \pm \\
4.3\end{array}$ & $\begin{array}{l}25.0 \pm \\
3.9\end{array}$ & $\begin{array}{l}\text { I 5; II } \\
\text { 15; } \\
\text { III 3; }\end{array}$ & $\begin{array}{l}\text { I 1; II } \\
21 ; \\
\text { III } 1 ;\end{array}$ & 8 & 14 & $\begin{array}{l}32 \pm \\
21\end{array}$ & $\begin{array}{l}33 \pm \\
16\end{array}$ & $4.3 \pm 1.4$ & $5.9 \pm 1.1$ & $\begin{array}{l}\text { I 5; II 8; III } \\
\text { 10; }\end{array}$ & $\begin{array}{l}\text { I 6; II 6; } \\
\text { III 11; }\end{array}$ & $\begin{array}{l}\text { CR 2; I 9; } \\
\text { II 3; III 9; }\end{array}$ & $\begin{array}{l}\text { CR 3; I 5; } \\
\text { II 5; III 10; }\end{array}$ & N/A & N/A & N/A & $\begin{array}{l}\mathrm{N} / \\
\mathrm{A}\end{array}$ \\
\hline $\begin{array}{l}\text { Chen } \\
2015 \\
{[20]}\end{array}$ & 50 & 100 & $\begin{array}{l}57.3 \pm \\
11.9\end{array}$ & $\begin{array}{l}58.3 \pm \\
11.3\end{array}$ & $\begin{array}{l}38 / \\
12\end{array}$ & $\begin{array}{l}76 / \\
24\end{array}$ & $\begin{array}{l}24.2 \pm \\
3.7\end{array}$ & $\begin{array}{l}24.6 \pm \\
3.1\end{array}$ & $\begin{array}{l}\text { I/II } \\
33 \\
\text { III } \\
17\end{array}$ & $\begin{array}{l}\text { I/II } \\
69 ; \\
\text { III } \\
31 ;\end{array}$ & 50 & 100 & $\begin{array}{l}22 \pm \\
15\end{array}$ & $\begin{array}{l}20 \pm \\
13\end{array}$ & $5.8 \pm 2.1$ & $6.7 \pm 2.0$ & II 20; III 30; & $\begin{array}{l}\text { II } 40 ; \\
\text { III } 60\end{array}$ & $\begin{array}{l}\text { CR 8; } \\
\text { I 13; II 12; } \\
\text { III 17; }\end{array}$ & $\begin{array}{l}\text { CR 17; } \\
\text { I 20; II 33; } \\
\text { III 30; }\end{array}$ & 8 & 18 & N/A & $\begin{array}{l}\mathrm{N} / \\
\mathrm{A}\end{array}$ \\
\hline $\begin{array}{l}\text { de' }^{\prime} \\
\text { Angeli } \\
\text { s 2015 } \\
{[21]}\end{array}$ & 32 & 32 & $\begin{array}{l}64.91 \\
\pm \\
10.05\end{array}$ & $\begin{array}{l}67.16 \\
\pm \\
9.61\end{array}$ & $\begin{array}{l}21 / \\
11\end{array}$ & $\begin{array}{l}21 / \\
11\end{array}$ & $\begin{array}{l}25.19 \\
\pm \\
3.52\end{array}$ & $\begin{array}{l}24.53 \\
\pm \\
3.19\end{array}$ & $\begin{array}{l}\text { I 21; } \\
\text { II 10; } \\
\text { III 1; }\end{array}$ & $\begin{array}{l}\text { I 18; } \\
\text { II 13; } \\
\text { III 1; }\end{array}$ & 27 & 23 & $\begin{array}{l}21.3 \\
8 \pm \\
5.57\end{array}$ & $\begin{array}{l}20.2 \\
5 \pm \\
5.70\end{array}$ & $\begin{array}{l}4 \\
(2.5-5)\end{array}$ & $\begin{array}{l}3.7 \\
(2.5-5)\end{array}$ & $\begin{array}{l}\text { T2 13; T3 } \\
\text { 17; T4 2; } \\
\text { N0 21; N1 } \\
\text { 10; N2 1; }\end{array}$ & $\begin{array}{l}\text { T2 16; } \\
\text { T3 13; } \\
\text { T4 3; N0 } \\
\text { 14; N1 15; } \\
\text { N2 3; }\end{array}$ & $\begin{array}{l}\text { CR 4; T1 3; } \\
\text { T2 12; } \\
\text { T3 11; } \\
\text { T4 2; N0 } \\
\text { 27; N1 5; } \\
\text { N2 0; }\end{array}$ & $\begin{array}{l}\text { CR 6; } \\
\text { T1 2; T2 9; } \\
\text { T3 13; } \\
\text { T4 2; N0 } \\
\text { 25; N1 6; } \\
\text { N2 1; }\end{array}$ & $\mathrm{N} / \mathrm{A}$ & N/A & $\begin{array}{l}32.06 \\
\pm \\
12.1\end{array}$ & $\begin{array}{l}62.9 \\
1 \pm \\
12.3\end{array}$ \\
\hline $\begin{array}{l}\text { Ferna } \\
\text { ndez- } \\
\text { Hevia } \\
2014 \\
{[22]}\end{array}$ & 37 & 37 & $\begin{array}{l}64.5 \pm \\
11.8\end{array}$ & $\begin{array}{l}69.5 \pm \\
10.5\end{array}$ & $\begin{array}{l}24 / \\
13\end{array}$ & $\begin{array}{l}22 / \\
15\end{array}$ & $\begin{array}{l}23.7 \pm \\
3.6\end{array}$ & $\begin{array}{l}25.1 \pm \\
4.0\end{array}$ & $\begin{array}{l}\text { I } 2 ; \\
\text { II } 28 ; \\
\text { III } 7 ;\end{array}$ & $\begin{array}{l}\text { I } 1 ; \text { II } \\
24 ; \\
\text { III } \\
12 ;\end{array}$ & 28 & 23 & $\begin{array}{l}26 \pm \\
14\end{array}$ & $\begin{array}{l}27 \pm \\
15\end{array}$ & $\begin{array}{l}\begin{array}{l}\text { Middle } \\
(\mathrm{n}=26)\end{array} \\
8.1 \pm 1.7 \\
\text { Low } \\
(\mathrm{n}=11) \\
3.5 \pm 1.2\end{array}$ & $\begin{array}{l}\begin{array}{l}\text { Middle } \\
(\mathrm{n}=24)\end{array} \\
8.2 \pm 1.5 \\
\text { Low } \\
(\mathrm{n}=13) \\
3.9 \pm 1.2\end{array}$ & $\begin{array}{l}\text { T2 8; T3 } \\
\text { 26; } \\
\text { T4 2; N0 } \\
\text { 22; } \\
\text { N1 10; } \\
\text { N2 4; M0 } \\
\text { 36; } \\
\text { M1 1; }\end{array}$ & $\begin{array}{l}\text { T2 10; } \\
\text { T3 21; } \\
\text { T4 3; N0 } \\
\text { 20; N1 10; } \\
\text { N2 3; } \\
\text { M0 33; } \\
\text { M1 4; }\end{array}$ & $\begin{array}{l}\text { CR 4; } \\
\text { T1 3; T2 7; } \\
\text { T3 22; } \\
\text { T4 1; N0 } \\
\text { 26; N1 8; } \\
\text { N2 3; }\end{array}$ & $\begin{array}{l}\text { CR 8; T1 1; } \\
\text { T2 7; } \\
\text { T3 16; T4 } \\
\text { 5; N0 31; } \\
\text { N1 5; N2 } \\
1 ;\end{array}$ & 6 & 10 & N/A & $\begin{array}{l}\mathrm{N} / \\
\mathrm{A}\end{array}$ \\
\hline $\begin{array}{l}\text { Lelon } \\
\text { g 2017 } \\
{[23]}\end{array}$ & 34 & 38 & N/A & N/A & $\begin{array}{l}23 / \\
11\end{array}$ & $\begin{array}{l}22 / \\
16\end{array}$ & $\begin{array}{l}24 \\
(18.6 \\
-45.0 \\
)\end{array}$ & $\begin{array}{l}24.2 \\
(17.7 \\
-32.7 \\
)\end{array}$ & $\begin{array}{l}\text { I } 6 ; \\
\text { II } 24 ; \\
\text { III } 4 ;\end{array}$ & $\begin{array}{l}\text { I 9; II } \\
27 ; \\
\text { III 2; }\end{array}$ & 30 & 35 & $\begin{array}{l}\mathrm{N} / \\
\mathrm{A}\end{array}$ & $\begin{array}{l}\mathrm{N} / \\
\mathrm{A}\end{array}$ & N/A & N/A & $\begin{array}{l}\text { T1 4; T2 4; } \\
\text { T3 24; } \\
\text { T4 2; N0 } \\
\text { 19; } \\
\text { N1 13; } \\
\text { M0 30; } \\
\text { M1 4; }\end{array}$ & $\begin{array}{l}\text { T1 2; T2 5; } \\
\text { T3 28; } \\
\text { T4 3; N0 } \\
\text { 18; N1 20; } \\
\text { M0 35; } \\
\text { M1 3; }\end{array}$ & $\begin{array}{l}\text { CR 7; } \\
\text { T1 3; T2 9; } \\
\text { T3 15; } \\
\text { T4 0; } \\
\text { N0 25; N1 } \\
7 ; \\
\text { N2 2; }\end{array}$ & $\begin{array}{l}\text { CR 12; } \\
\text { T1 5; T2 } \\
\text { 10; T3 10; } \\
\text { T4 1; N0 } \\
\text { 33; N1 5; } \\
\text { N2 0; }\end{array}$ & 19 & 21 & $\begin{array}{l}31.9 \\
(29.3 \\
-42)\end{array}$ & $\begin{array}{l}53.3 \\
(8-95 \\
)\end{array}$ \\
\hline $\begin{array}{l}\text { Perda } \\
\text { wood } \\
2015 \\
{[24]}\end{array}$ & 25 & 25 & $\begin{array}{l}70 \\
(54-7 \\
6)\end{array}$ & $\begin{array}{l}70 \\
(49-8 \\
4)\end{array}$ & $\begin{array}{l}19 / \\
6\end{array}$ & $\begin{array}{l}19 / \\
6\end{array}$ & $\begin{array}{l}28 \\
(18- \\
46)\end{array}$ & $\begin{array}{l}26 \\
(19- \\
38)\end{array}$ & $\begin{array}{l}\text { I } 5 ; \\
\text { II 14; } \\
\text { III 6; }\end{array}$ & $\begin{array}{l}\text { I } 8 ; \text { II } \\
14 ; \\
\text { III 3; }\end{array}$ & 7 & 4 & $\begin{array}{l}50 \\
(20- \\
70)\end{array}$ & $\begin{array}{l}50 \\
(20- \\
80)\end{array}$ & $\begin{array}{l}8 \\
(4-10)\end{array}$ & $\begin{array}{l}8 \\
(5-10)\end{array}$ & $\begin{array}{l}\text { T2 4; T3 } \\
\text { 19; } \\
\text { T4 2; N0 } \\
\text { 18; } \\
\text { N1 4; } \\
\text { N2 3; } \\
\text { M0 22; } \\
\text { M1 3; }\end{array}$ & $\begin{array}{l}\text { T2 7; } \\
\text { T3 18; } \\
\text { T4 0; N0 } \\
\text { 11; N1 5; } \\
\text { N2 9;M0 } \\
\text { 24; M1 1; }\end{array}$ & $\begin{array}{l}\text { T0 4; T1 8; } \\
\text { T2 36; } \\
\text { T3 48; T4 } \\
\text { 4; N0 69; } \\
\text { N1 19; N2 } \\
\text { 12; }\end{array}$ & $\begin{array}{l}\text { T0 4; T1 2; } \\
\text { T2 33; } \\
\text { T3 54; T4 } \\
\text { 7; N0 67; } \\
\text { N1 20; N2 } \\
\text { 13; }\end{array}$ & 6 & 5 & N/A & $\begin{array}{l}\mathrm{N} / \\
\mathrm{A}\end{array}$ \\
\hline $\begin{array}{l}\text { Perda } \\
\text { wood } \\
2017 \\
{[25]}\end{array}$ & 100 & 100 & $\begin{array}{l}67.33 \\
\pm \\
10.80 \\
7\end{array}$ & $\begin{array}{l}66.86 \\
\pm \\
10.73 \\
3\end{array}$ & $\begin{array}{l}72 / \\
28\end{array}$ & $\begin{array}{l}69 / \\
31\end{array}$ & $\begin{array}{l}25.65 \\
\pm \\
3.924\end{array}$ & $\begin{array}{l}25.43 \\
\pm \\
4.437\end{array}$ & $\begin{array}{l}\text { I } 41 ; \\
\text { II 39; } \\
\text { III } \\
20\end{array}$ & $\begin{array}{l}\text { I 36; } \\
\text { II 53; } \\
\text { III } \\
\text { 11; }\end{array}$ & 18 & 27 & $\begin{array}{l}\mathrm{N} / \\
\mathrm{A}\end{array}$ & $\begin{array}{l}\mathrm{N} / \\
\mathrm{A}\end{array}$ & $\begin{array}{l}7.53 \pm \\
1.972\end{array}$ & $\begin{array}{l}7.83 \pm \\
1.781\end{array}$ & $\begin{array}{l}\text { T2 56; T3 } \\
\text { 43; } \\
\text { T4 1; N0 } \\
\text { 81; } \\
\text { N1 8; } \\
\text { N2 11; } \\
\text { M0 94; M1 } \\
6 ;\end{array}$ & $\begin{array}{l}\text { T2 45; } \\
\text { T3 53; } \\
\text { T4 2; N0 } \\
\text { 34; } \\
\text { N1 23; } \\
\text { N2 43; } \\
\text { M0 91; } \\
\text { M1 9; }\end{array}$ & $\begin{array}{l}\text { T0 0; T1 0; } \\
\text { T2 8; } \\
\text { T3 16; T4 } \\
\text { 1; N0 14; } \\
\text { N1 8; N2 } \\
\text { 3; }\end{array}$ & $\begin{array}{l}\text { T0 1; } \\
\text { T1 1; } \\
\text { T2 4; } \\
\text { T3 18; } \\
\text { T4 1; N0 } \\
\text { 14; N1 5; } \\
\text { N2 6; }\end{array}$ & 23 & 23 & $\mathrm{~N} / \mathrm{A}$ & $\begin{array}{l}\mathrm{N} / \\
\mathrm{A}\end{array}$ \\
\hline $\begin{array}{l}\text { Rasulo } \\
\text { v } 2016 \\
\text { [26] }\end{array}$ & 22 & 23 & $\begin{array}{l}56 \\
(30-6 \\
9)\end{array}$ & $\begin{array}{l}60 \\
(15-7 \\
8)\end{array}$ & $\begin{array}{l}11 / \\
11\end{array}$ & $\begin{array}{l}14 / \\
9\end{array}$ & $\begin{array}{l}26.0 \\
(19.7 \\
-32.3 \\
)\end{array}$ & $\begin{array}{l}26.0 \\
(18.3 \\
-37.2 \\
)\end{array}$ & N/A & N/A & 19 & 11 & $\begin{array}{l}\mathrm{N} / \\
\mathrm{A}\end{array}$ & $\begin{array}{l}\mathrm{N} / \\
\mathrm{A}\end{array}$ & $\begin{array}{l}\text { Median } \\
6.5\end{array}$ & $\begin{array}{l}\text { Median } \\
7\end{array}$ & $\begin{array}{l}\text { T1-2 5; T3 } \\
\text { 14; } \\
\text { T4a 3; N0 } \\
\text { 2; } \\
\text { N1-2 20; }\end{array}$ & $\begin{array}{l}\text { T1-2 7; } \\
\text { T3 14; T4a } \\
\text { 2; N0 5; } \\
\text { N1-2 18; }\end{array}$ & $\begin{array}{l}\text { T0 6; T1-2 } \\
\text { 11; } \\
\text { T3 5; T4a } \\
\text { 0; N0 16; } \\
\text { N1-2 6; }\end{array}$ & $\begin{array}{l}\text { T0 2; T1-2 } \\
\text { 12; } \\
\text { T3 9; T4a } \\
0 ; \text { N0 19; } \\
\text { N1-2 4; }\end{array}$ & N/A & N/A & N/A & $\begin{array}{l}\mathrm{N} / \\
\mathrm{A}\end{array}$ \\
\hline $\begin{array}{l}\text { Velthu } \\
\text { is } 2014 \\
\text { [27] }\end{array}$ & 25 & 25 & $\begin{array}{l}\text { Mean } \\
64, \\
\text { Rang } \\
\text { e } \\
49-86\end{array}$ & $\begin{array}{l}\text { Mea } \\
\text { n 65, } \\
\text { Rang } \\
\text { e } \\
38-81\end{array}$ & $\begin{array}{l}18 / \\
7\end{array}$ & $\begin{array}{l}18 / \\
7\end{array}$ & $\begin{array}{l}\text { Mea } \\
\text { n 25, } \\
\text { Medi } \\
\text { an } \\
\text { (Ran } \\
\text { ge) } \\
25 \\
\text { (20-3 } \\
6)\end{array}$ & $\begin{array}{l}\text { Mea } \\
\mathrm{n} 27, \\
\text { Medi } \\
\text { an } \\
\text { (Ran } \\
\text { ge) } \\
28 \\
(21-3 \\
4)\end{array}$ & N/A & N/A & 25 & 25 & $\begin{array}{l}\text { Me } \\
\text { an } \\
33, \\
\text { Ran } \\
\text { ge } \\
18- \\
90\end{array}$ & $\begin{array}{l}\text { Me } \\
\text { an } \\
33, \\
\text { Ran } \\
\text { ge } \\
9-6 \\
0\end{array}$ & $\begin{array}{l}\text { Mean 8, } \\
\text { Median } \\
\text { (Range) } \\
8(0-16)\end{array}$ & $\begin{array}{l}\text { Mean 6, } \\
\text { Median } \\
\text { (Range) } \\
7(0-15)\end{array}$ & $\begin{array}{l}\text { T1 1; T2 } \\
\text { 11; } \\
\text { T3 13; } \\
\text { N0 17; } \\
\text { N1 5; N2 3; }\end{array}$ & $\begin{array}{l}\text { T1 1; T2 9; } \\
\text { T3 15; } \\
\text { N0 15; N1 } \\
\text { 8; N2 2; }\end{array}$ & N/A & $\mathrm{N} / \mathrm{A}$ & No & No & N/A & $\begin{array}{l}\mathrm{N} / \\
\mathrm{A}\end{array}$ \\
\hline
\end{tabular}

S.D. : standard deviation; BMI : body mass index; ASA : American Society of Anaesthesiology; CR : complete remission; N/A : not available; Ta : transanal total mesorectal excision; La : laparoscopic total mesorectal excision. Italics indicate statistically significant difference $(P<0.05)$

The short-term postoperative outcomes are shown in Figure 4, Supplementary Figures S6, S7, and Table 4. Seven studies [19-24, 26] investigated the overall postoperative complications, among which significant fewer complications were observed in the TaTME group (RR 0.74, 95\%CI 0.58-0.95, $P=0.02$ ). Three studies [21, 22, 26] graded the postoperative complications according to the Dindo-Clavien classification [30]. When compared separately, however, no difference was found for any Dindo-Clavien grade. Regarding the individual postoperative complica- tions, anastomotic leakage [19-22, 24-26], prolonged postoperative ileus [19-22, 24, 26], and wound infection [20, 21, 24-26] were found comparable between the groups. Patients undergoing TaTME tended to have a lower incidence of urinary retention [20-22, 24-26]; however, the difference failed to reach significance $(P=0.05)$. The early postoperative mortality was studied by $4[19,21,25,26]$, among which only in the study by Perdawood and colleagues [25] were 2 and 4 death reported in the TaTME and LaTME groups, respectively. In addition, less 
readmission was associated with TaTME (RR 0.51, 95\% CI 0.33-0.79, $P=0.003$ ) [20-25].

Finally, publication bias was evaluated graphically using funnel plots, as some are shown in Figure
5. As there was no evidence of significant publication bias, the pooled analyses results were considered reliable.

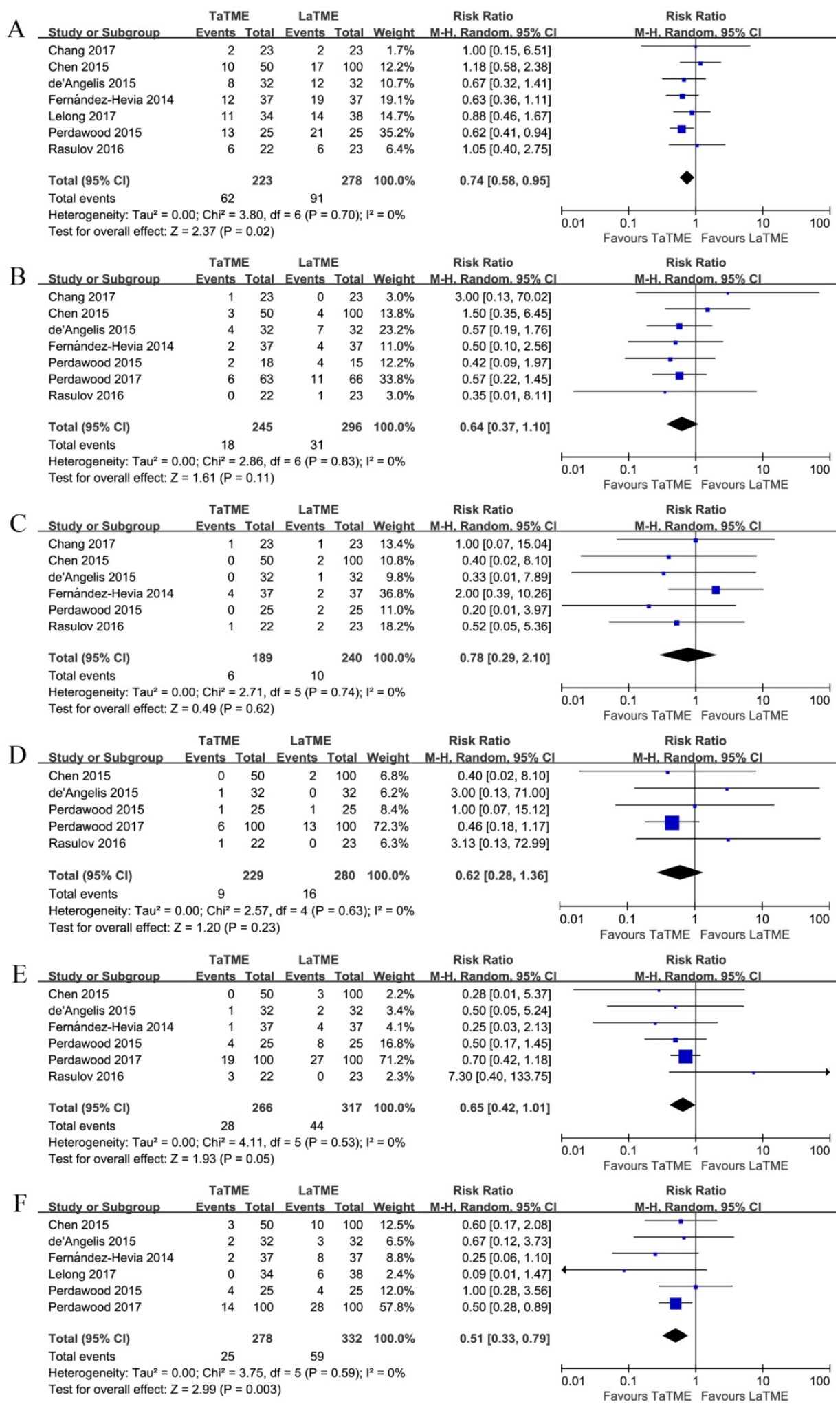

Figure 4. Forest plots of risk ratios of short-term postoperative outcomes of the included studies in random-effects model, (A) overall postoperative complications, (B) anastomotic leakage, $(C)$ prolonged postoperative ileus, $(D)$ wound infection, $(E)$ urinary retention, and $(F)$ readmission. TaTME : transanal total mesorectal excision; LaTME : laparoscopic total mesorectal excision; $\mathrm{M}-\mathrm{H}$ : Mantel-Haenszel; $\mathrm{Cl}$ : confidence interval. 

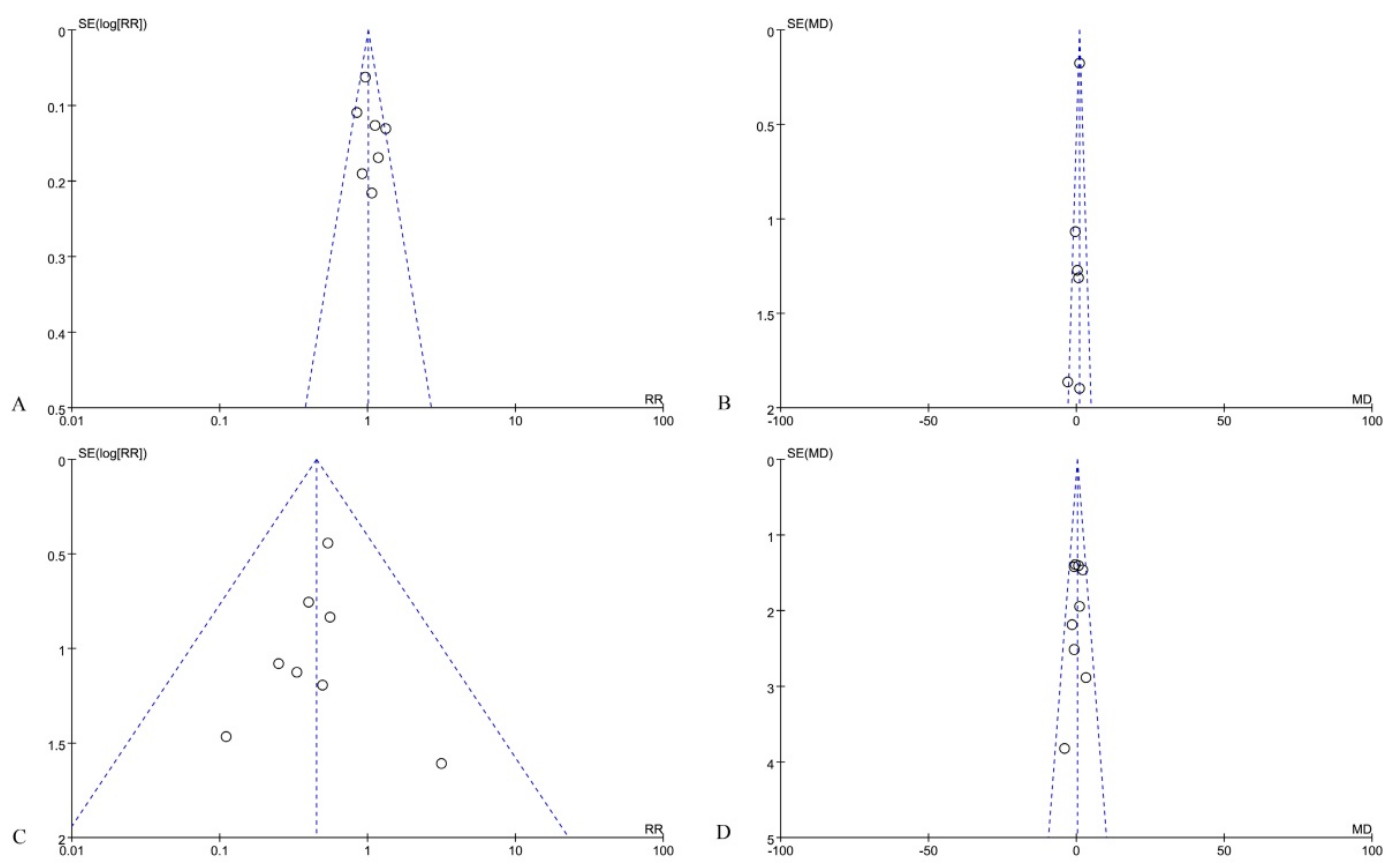

Figure 5. Funnel plots of risk ratios and mean differences of oncological outcomes of the included studies, (A) Quirke quality of mesorectum to be complete, (B) circumferential resection margin, (C) positive circumferential resection margin, and (D) harvested lymph nodes.

Table 4. Results of oncological, perioperative and postoperative outcomes of the included studies.

\begin{tabular}{|c|c|c|c|c|c|c|c|c|c|c|c|}
\hline Outcomes & $\begin{array}{l}\text { Number of } \\
\text { studies }\end{array}$ & Number of patients & $\begin{array}{l}\text { RR or MD } \\
\text { (random) }\end{array}$ & $\begin{array}{l}95 \% \mathrm{CI} \\
\text { (random) }\end{array}$ & $\begin{array}{l}P \\
\text { (random) }\end{array}$ & $\begin{array}{l}\text { RR or MD } \\
\text { (fixed) }\end{array}$ & $\begin{array}{l}95 \% \mathrm{CI} \\
\text { (fixed) }\end{array}$ & $\begin{array}{l}P \\
\text { (fixed) }\end{array}$ & $\mathbf{I}^{2}$ & $\mathrm{Chi}^{2}$ & $\begin{array}{l}P \\
\left(\mathrm{Chi}^{2}\right)\end{array}$ \\
\hline \multicolumn{12}{|c|}{ Quirke quality of mesorectum } \\
\hline Complete & 7 & TaTME 275 LaTME 280 & 1.03 & {$[0.92,1.16]$} & 0.57 & 1.00 & {$[0.91,1.11]$} & 0.93 & $34 \%$ & 9.10 & 0.17 \\
\hline Nearly complete & 7 & TaTME 275 LaTME 280 & 1.35 & {$[0.95,1.91]$} & 0.10 & 1.41 & {$[0.99,2.00]$} & 0.05 & $0 \%$ & 5.46 & 0.49 \\
\hline Incomplete & 7 & TaTME 275 LaTME 280 & 0.64 & {$[0.39,1.06]$} & 0.08 & 0.57 & {$[0.35,0.91]$} & 0.02 & $0 \%$ & 5.64 & 0.47 \\
\hline CRM & 6 & TaTME 269 LaTME 319 & $0.64^{*}$ & {$[-0.09,1.37]$} & 0.08 & $0.92 *$ & {$[0.58,1.25]$} & $<0.00001$ & $15 \%$ & 5.89 & 0.32 \\
\hline \multicolumn{12}{|l|}{ Positive CRM } \\
\hline Overall & 9 & TaTME 348 LaTME 403 & 0.47 & {$[0.26,0.84]$} & 0.01 & 0.45 & {$[0.26,0.79]$} & 0.005 & $0 \%$ & 3.00 & 0.88 \\
\hline$<1 \mathrm{~mm}$ & 7 & TaTME 291 LaTME 346 & 0.48 & {$[0.26,0.89]$} & 0.02 & 0.46 & {$[0.25,0.83]$} & 0.01 & $0 \%$ & 2.91 & 0.71 \\
\hline DRM & 7 & TaTME 292 LaTME 342 & $0.34^{*}$ & {$[-0.09,0.78]$} & 0.12 & - & - & - & $75 \%$ & 24.00 & 0.0005 \\
\hline Positive DRM & 4 & TaTME 189 LaTME 193 & 0.71 & {$[0.15,3.40]$} & 0.67 & 0.82 & {$[0.22,3.02]$} & 0.76 & $0 \%$ & 2.26 & 0.52 \\
\hline Harvested lymph nodes & 9 & TaTME 348 LaTME 403 & $0.21^{*}$ & {$[-0.96,1.38]$} & 0.72 & $0.21^{*}$ & {$[-0.96,1.38]$} & 0.72 & $0 \%$ & 5.49 & 0.70 \\
\hline Operative time & 8 & TaTME 323 LaTME 378 & $-19.76^{*}$ & {$[-39.44,-0.07]$} & 0.05 & - & - & - & $77 \%$ & 30.45 & $<0.0001$ \\
\hline One team in TaTME & 5 & TaTME 214 LaTME 218 & $-32.06^{*}$ & {$[-50.33,-13.80]$} & 0.0006 & - & - & - & $49 \%$ & 7.84 & 0.10 \\
\hline Blood loss & 5 & TaTME 220 LaTME 271 & $-66.78^{*}$ & {$[-124.18,-9.38]$} & 0.02 & - & - & - & $80 \%$ & 19.79 & 0.0005 \\
\hline Conversion & 8 & TaTME 323 LaTME 378 & 0.25 & {$[0.09,0.68]$} & 0.007 & 0.18 & {$[0.07,0.45]$} & 0.0003 & $0 \%$ & 4.88 & 0.43 \\
\hline Diverting Ostomy & 6 & TaTME 198 LaTME 253 & 1.00 & {$[0.97,1.04]$} & 0.86 & 1.01 & {$[0.96,1.07]$} & 0.62 & $0 \%$ & 3.42 & 0.64 \\
\hline $\begin{array}{l}\text { Splenic flexure } \\
\text { mobilization }\end{array}$ & 4 & TaTME 212 LaTME 262 & 1.64 & {$[1.19,2.27]$} & 0.003 & 1.65 & {$[1.22,2.24]$} & 0.001 & $10 \%$ & 3.35 & 0.34 \\
\hline \multicolumn{12}{|c|}{ Intraoperative complications } \\
\hline Overall & 5 & TaTME 244 LaTME 294 & 1.04 & {$[0.57,1.90]$} & 0.90 & 1.04 & {$[0.57,1.90]$} & 0.91 & $0 \%$ & 0.32 & 0.85 \\
\hline Intraoperative bleeding & 3 & TaTME 175 LaTME 225 & 2.39 & {$[0.85,6.71]$} & 0.10 & 2.53 & {$[0.92,6.95]$} & 0.07 & $0 \%$ & 0.90 & 0.64 \\
\hline Adjacent organ injury & 3 & TaTME 172 LaTME 223 & 1.51 & {$[0.41,5.49]$} & 0.53 & 1.53 & {$[0.43,5.47]$} & 0.51 & $0 \%$ & 0.32 & 0.85 \\
\hline Hospital stay & 8 & TaTME 323 LaTME 378 & $-1.25^{*}$ & {$[-2.57,0.07]$} & 0.06 & - & - & - & $70 \%$ & 23.23 & 0.002 \\
\hline Hospital stay no ERAS & 3 & TaTME 162 LaTME 162 & $-4.23^{*}$ & {$[-7.01,-1.45]$} & 0.003 & - & - & - & $42 \%$ & 3.47 & 0.18 \\
\hline \multicolumn{12}{|c|}{ Postoperative complications } \\
\hline Overall & 7 & TaTME 223 LaTME 278 & 0.74 & {$[0.58,0.95]$} & 0.02 & 0.78 & {$[0.61,1.01]$} & 0.06 & $0 \%$ & 3.80 & 0.70 \\
\hline Dindo-Clavien I & 3 & TaTME 91 LaTME 92 & 0.98 & {$[0.50,1.92]$} & 0.95 & 1.01 & {$[0.52,1.96]$} & 0.98 & $0 \%$ & 0.78 & 0.68 \\
\hline Dindo-Clavien II & 3 & TaTME 91 LaTME 92 & 0.68 & {$[0.27,1.76]$} & 0.43 & 0.65 & {$[0.26,1.59]$} & 0.34 & $0 \%$ & 1.82 & 0.40 \\
\hline Dindo-Clavien III & 3 & TaTME 91 LaTME 92 & 0.55 & {$[0.18,1.69]$} & 0.30 & 0.53 & {$[0.18,1.61]$} & 0.26 & $0 \%$ & 0.46 & 0.79 \\
\hline Dindo-Clavien IV & 3 & TaTME 91 LaTME 92 & 0.41 & {$[0.08,2.06]$} & 0.28 & 0.40 & {$[0.08,2.03]$} & 0.27 & $0 \%$ & 0.05 & 0.97 \\
\hline Anastomotic leakage & 7 & TaTME 245 LaTME 296 & 0.64 & {$[0.37,1.10]$} & 0.11 & 0.65 & {$[0.38,1.10]$} & 0.11 & $0 \%$ & 2.86 & 0.83 \\
\hline Prolonged ileus & 6 & TaTME 189 LaTME 240 & 0.78 & {$[0.29,2.10]$} & 0.62 & 0.72 & {$[0.29,1.80]$} & 0.49 & $0 \%$ & 2.71 & 0.74 \\
\hline Wound infection & 5 & TaTME 229 LaTME 280 & 0.62 & {$[0.28,1.36]$} & 0.23 & 0.64 & {$[0.30,1.35]$} & 0.24 & $0 \%$ & 2.57 & 0.63 \\
\hline Urinary retention & 6 & TaTME 266 LaTME 317 & 0.65 & {$[0.42,1.01]$} & 0.05 & 0.67 & {$[0.44,1.02]$} & 0.06 & $0 \%$ & 4.11 & 0.53 \\
\hline Readmission & 6 & TaTME 278 LaTME 332 & 0.51 & {$[0.33,0.79]$} & 0.003 & 0.48 & {$[0.31,0.73]$} & 0.0008 & $0 \%$ & 3.75 & 0.59 \\
\hline
\end{tabular}

RR : risk ratio; $\mathrm{MD}$ : mean difference; $\mathrm{CI}$ : confidence interval; CRM : circumferential resection margin; DRM : distal resection margin; ERAS : enhanced recovery after surgery; TaTME : transanal total mesorectal excision; LaTME : laparoscopic total mesorectal excision. Asterisks $\left({ }^{*}\right)$ indicate mean differences, whereas others are risk ratios. Italics indicate statistically significant difference $(P<0.05)$ 


\section{Discussion}

The present meta-analysis investigated the novel bottom-up procedure of transanal total mesorectal excision (TaTME) in comparison with the conventional laparoscopic TME surgery, in terms of oncological outcomes, as well as perioperative and postoperative outcomes. In the pooled analysis of 9 retrospective cohort studies, we demonstrated less involved CRM in patients undergoing TaTME compared with LaTME, whereas the quality of mesorectum, CRM, DRM, and harvested lymph nodes were comparable. Regarding the perioperative outcomes, we found shorter operative time, less blood loss, less conversion rate, and shorter hospital stay associated with TaTME; however, the intraoperative complications were similar. Finally, the postoperative complications were mostly comparable between the groups, though overall postoperative complications and readmission were less in patients receiving TaTME. Therefore, it appears that TaTME achieved a better resection quality and smoother recovery, without compromising the short-term safety.

Laparoscopic surgery for rectal cancer is a well-established technique and has been widely accepted by surgeons as it shows in some randomized trials a certain degree of benefits with respect to postoperative recovery [3-7, 31, 32]. Nevertheless, in two most recent randomized trials the laparoscopic surgery failed to achieve noninferiority for pathological outcomes compared with open resection, although individual oncological results were similar $[33,34]$. The assessment of noninferiority in the randomized trials is based on a combination of the completeness of mesorectum, circumferential and distal resection margins. In the present study comparing TaTME with LaTME, it was impossible to investigate the combined result of pathological quality from the included studies; however, less positive CRM was observed associated with TaTME, along with comparable results regarding completeness of mesorectum, CRM, and DRM, which might contribute to a better surgical quality concerning the combined result.

TaTME was developed to overcome the difficulties encountered in the practice of laparoscopic surgery, with the use of dedicated transanal platforms to secure a precise dissection around the mesorectum. The DRM can be determined under direct visualization, and $\mathrm{CO}_{2}$ insufflation could facilitate dissection within the avascular plane around the perirectal fascia. Thus, TaTME is potentially beneficial to maintain an uninvolved CRM and intact mesorectum, as well as a safe distance of DRM. However, with the theoretical advantages in favour of TaTME, the completeness of mesorectum and DRM were not superior to LaTME. One explanation is that many of the studies included were performed by teams with initial experience of this novel technique, although they are usually previously dedicated to laparoscopic and colorectal surgery; whereas, the laparoscopic procedure is relatively well-established. Besides, the analysis of DRM could be biased because of the heterogeneity of the tumour location from anal verge. Additionally, pelvic tissue fibrosis after preoperative radiation may result in underestimated circumferential and distal margins. The CRM involvement is a major prognostic factor related to locoregional recurrence [35]. Most of the included studies of this meta-analysis defined a positive CRM as tumour cells found within $1 \mathrm{~mm}$ from the resection margin, which is widely accepted by most studies; however, the study of Nagtegaal and colleagues [36] demonstrates that an at least $2 \mathrm{~mm} \mathrm{CRM}$ is significantly safer, and the 2-year local recurrence of patients with a CRM between 1 and $2 \mathrm{~mm}$ is comparable with that $<1 \mathrm{~mm}$. Thus, the benefits in CRM involvement found in the present study should be carefully interpreted.

The long-term oncological outcomes are the most important factors to be evaluated after surgery with cancer patients; nevertheless, they are currently impossible to determine. Two included studies in this systematic review reported comparable long-term outcomes in terms of recurrence and 2-year survival $[21,23]$. However, the patient population was too small to draw any conclusions, and 2-year survival is not long enough for a proper survival evaluation. A recent study by Mark and colleagues [37], who performed their TME surgery with a combination of transanal and transabdominal approaches in 373 patients, reports a promising 5-year actuarial survival as $90 \%$. This is similar to the 3-year survival reported in the randomized trials of laparoscopic surgery [3, 31]. However, the procedure Marks and colleagues performed is not an actual TaTME, so the long-term outcomes of TaTME in direct comparison with LaTME should be awaited.

The major advantages of TaTME lied in the operative and recovery results. The operative time was shorter in TaTME, which is a disadvantage of the laparoscopic surgery $[5,6]$. The shorter operative time may be due to the convenience of dissecting the mesorectum from below under adequate visualization. Besides, it is possible to perform the transabdominal and transanal parts of procedure simultaneously by two teams, which could significantly shorten the operative time [20]. Interestingly, however, we identified a shorter operative time when only one-team TaTME was included, but the overall comparison did not reach significance. One explanation is that the definitions of operative time 
were obscure, and the time for instrument set-up cannot be overlooked, but only in the study of Perdawood and colleagues [25] was the definition of operative time clearly stated as from incision to stich. In addition, many teams may be in the middle of the learning curve, where TaTME was performed slower than it should be in experienced hands. Conversion is a major problem in laparoscopic rectal surgery, which is frequently needed in male, obese patients, or in case of distantly located or bulky tumours [38]. The reported conversion rate is as high as $34 \%$ in the CLASICC trial [7], which results in more complications and worse outcomes. Conversion is mainly caused by fixity or inaccessibility of the tumour, and uncertainty of tumour clearance and anatomy, where the transanal approach could provide additional advantages during the dissection of complicate pelvis. The present meta-analysis showed a less conversion rate associated with TaTME; however, the nonrandomized retrospective trials without intention-totreat design could result in considerable selection bias. The hospital stay was shorter in patients undergoing TaTME. The faster recovery identified in the present study may be the reason of less postoperative analgesia from less wound trauma in patients undergoing TaTME, as the specimen could be extracted transanally without the need of a Pfannenstiel incision. Besides, less conversion is also associated with shorter hospital stay [7]. However, the definitions of the hospital stay in the included studies were not clear, with only 4 defined it as post-operative $[19,25-27]$; and the postoperative recovery strategies and criteria to discharge were heterogeneous, with some implemented ERAS [20] and others did not.

The two surgical procedures showed comparable intraoperative and postoperative complications, with the postoperative complications appeared slightly less in patients undergoing TaTME. Anastomotic leakage is a major concern after surgery with gastrointestinal anastomosis. In laparoscopic surgery, multiple liner stapler firings are required as the limitations of instrumentation within the narrow deep pelvic cavity, which are associated with anastomotic leakage [39]. The novel TaTME technique may overcome this issue, as the use of liner staplers could be avoided; however, the anastomotic leakage between TaTME and LaTME procedure was comparable in the present meta-analysis. This may be due to the prolonged perineal operative time in TaTME, as indicated by Penna and colleagues [40], offsetting its benefits.

Nevertheless, there are still some concerns about this novel technique. TaTME is approached from the anorectal lumen to the perirectal space; although the rectal lumen is routinely closed by using a purse- string suture and rinsed with antiseptic solution, there is still a potential risk of tumour cell dissemination and bacterial contamination $[13,14]$. Besides, there are some new serious complications documented, such as urethral injury, and pelvic side wall injury with bleeding and nerve damage, which are especially associated with transanal surgery [5, 6, 11, 41]. Whereas, the studies included in the present systematic review did not document such issues, mostly because of the small size of the patient population. Furthermore, two included studies [21, 23] in the present systematic review showed comparable postoperative anal sphincter function; however, there is a potential risk of damaging the anal sphincters, since a fixed retractor and a transanal surgical platform have to be inserted into the anal canal for the entire endoscopic transanal dissection. Although some literature shows the safety of these devices when applied to the transanal endoscopic microsurgery [42], the duration of a radical surgery takes significantly longer. Additionally, Chi and colleagues have recently proposed an anatomical aspect of view that the standard of TME is probably not achieved in some TaTME trials since the bowel transection planes are usually below standard in those mid and low rectal cancer cases [43].

Several limitations must be taken into consideration when viewing the present systematic review and meta-analysis. The main limitation is the included studies were not randomized clinical trials, but retrospective cohort studies, with relatively lowquality evidence and considerable heterogeneity. Besides, the long-term oncological outcomes were not available in most of the studies. Two multicentre randomized trials, COLOR III and GRECCAR 11, are currently ongoing $[44,45]$, which will provide the best-quality evidence regarding the potential benefits and pitfalls in short- and long-term, as well as determine the indications of this novel technique. In addition, the novel transanal procedure was compared with the well-established transabdominal laparoscopic procedure, without any quality assurance process; and only one study was propensity score matched between groups [25], where some of them were not even matched at all [22, 23, 26]. Furthermore, the patient inclusion and exclusion criteria were heterogeneous among the studies, as some studies included the locally advanced tumours and even stage IV patients. Thus, caution must be exercised when interpreting the results of this meta-analysis.

The present systematic review and meta-analysis suggested some advantages of TaTME, in terms of circumferential resection margin involvement, operative time, blood loss, conversion, hospital stay, 
overall postoperative complications, and readmission. It appears that the TaTME procedure achieved a better resection quality and smoother recovery in selected patients, without compromising the shortterm safety. We provided a critical review of the currently available literature, neutrally evaluating its benefits and pitfalls. The TaTME technique is implemented at its earliest stage; it is too early to conclude that the TaTME is superior to laparoscopic surgery, since results of large randomized studies with adequate duration of follow-up have to be awaited. Training programmes have been proposed to accredit surgeons in safely implementing TaTME with appropriate patient selection and quality assurance $[46,47]$. As it is technically demanding, the procedure needs to be taught and learned systematically with appropriate cadaver courses and mentorship to ensure safe implementation in high-volume centers.

\section{Supplementary Material}

Supplementary figures.

http://www.jcancer.org/v10p0341s1.pdf

\section{Competing Interests}

The authors have declared that no competing interest exists.

\section{References}

1. Ferlay J, Soerjomataram I, Ervik M, Dikshit R, Eser S, Mathers C, et al. GLOBOCAN 2012 v1.0, Cancer Incidence and Mortality Worldwide: IARC CancerBase No. 11 [Internet]. Lyon, France: International Agency for Research on Cancer; 2013.

2. Heald RJ, Ryall RD. Recurrence and survival after total mesorectal excision for rectal cancer. Lancet (London, England). 1986; 1: 1479-82.

3. Bonjer HJ, Deijen CL, Abis GA, Cuesta MA, van der Pas MH, de Lange-de Klerk ES, et al. A randomized trial of laparoscopic versus open surgery for rectal cancer. The New England journal of medicine. 2015; 372: 1324-32.

4. Jayne DG, Guillou PJ, Thorpe H, Quirke P, Copeland J, Smith AM, et al. Randomized trial of laparoscopic-assisted resection of colorectal carcinoma: 3-year results of the UK MRC CLASICC Trial Group. Journal of clinical oncology : official journal of the American Society of Clinical Oncology. 2007; 25: 3061-8.

5. Kang SB, Park JW, Jeong SY, Nam BH, Choi HS, Kim DW, et al. Open versus laparoscopic surgery for mid or low rectal cancer after neoadjuvant chemoradiotherapy (COREAN trial): short-term outcomes of an open-label randomised controlled trial. The Lancet Oncology. 2010; 11: 637-45.

6. van der Pas MH, Haglind E, Cuesta MA, Furst A, Lacy AM, Hop WC, et al. Laparoscopic versus open surgery for rectal cancer (COLOR II): short-term outcomes of a randomised, phase 3 trial. The Lancet Oncology. 2013; 14: 210-8.

7. Guillou PJ, Quirke P, Thorpe H, Walker J, Jayne DG, Smith AM, et al. Short-term endpoints of conventional versus laparoscopic-assisted surgery in patients with colorectal cancer (MRC CLASICC trial): multicentre, randomised controlled trial. Lancet (London, England). 2005; 365: 1718-26.

8. Whiteford MH, Denk PM, Swanstrom LL. Feasibility of radical sigmoid colectomy performed as natural orifice translumenal endoscopic surgery (NOTES) using transanal endoscopic microsurgery. Surgical endoscopy. 2007; 21: $1870-4$.

9. Sylla P, Rattner DW, Delgado S, Lacy AM. NOTES transanal rectal cancer resection using transanal endoscopic microsurgery and laparoscopic assistance. Surgical endoscopy. 2010; 24: 1205-10.

10. Pellino G, Warusavitarne J. Medium-term adoption trends for laparoscopic, robotic and transanal total mesorectal excision (TaTME) techniques. Techniques in coloproctology. 2017; 21: 911-3.

11. Penna M, Hompes R, Arnold S, Wynn G, Austin R, Warusavitarne J, et al. Transanal Total Mesorectal Excision: International Registry Results of the First 720 Cases. Annals of surgery. 2017; 266: 111-7.
12. Veltcamp Helbach M, Deijen CL, Velthuis S, Bonjer HJ, Tuynman JB, Sietses C. Transanal total mesorectal excision for rectal carcinoma: short-term outcomes and experience after 80 cases. Surgical endoscopy. 2016; 30: 464-70.

13. Deijen CL, Tsai A, Koedam TW, Veltcamp Helbach M, Sietses C, Lacy AM, et al. Clinical outcomes and case volume effect of transanal total mesorectal excision for rectal cancer: a systematic review. Techniques in coloproctology. 2016; 20: 811-24.

14. Velthuis S, Veltcamp Helbach M, Tuynman JB, Le TN, Bonjer HJ, Sietses C. Intra-abdominal bacterial contamination in TAMIS total mesorectal excision for rectal carcinoma: a prospective study. Surgical endoscopy. 2015; 29: 3319-23

15. Moher D, Liberati A, Tetzlaff J, Altman DG. Preferred reporting items for systematic reviews and meta-analyses: The PRISMA statement. International Journal of Surgery. 2010; 8: 336-41.

16. Stang A. Critical evaluation of the Newcastle-Ottawa scale for the assessment of the quality of nonrandomized studies in meta-analyses. European journal of epidemiology. 2010; 25: 603-5.

17. Hozo SP, Djulbegovic B, Hozo I. Estimating the mean and variance from the median, range, and the size of a sample. BMC medical research methodology. 2005; 5: 13 .

18. Cochrane Handbook for Systematic Reviews of Interventions. In. The Cochrane Collaboration. 1999. http:/ / handbook.cochrane.org.

19. Chang TC, Kiu KT. Transanal Total Mesorectal Excision in Lower Rectal Cancer: Comparison of Short-Term Outcomes with Conventional Laparoscopic Total Mesorectal Excision. Journal of laparoendoscopic \& advanced surgical techniques Part A. 2017.

20. Chen CC, Lai YL, Jiang JK, Chu CH, Huang IP, Chen WS, et al. Transanal Total Mesorectal Excision Versus Laparoscopic Surgery for Rectal Cancer Receiving Neoadjuvant Chemoradiation: A Matched Case-Control Study. Annals of surgical oncology. 2016; 23: 1169-76.

21. de'Angelis N, Portigliotti L, Azoulay D, Brunetti F. Transanal total mesorectal excision for rectal cancer: a single center experience and systematic review of the literature. Langenbeck's archives of surgery. 2015; 400: 945-59.

22. Fernandez-Hevia M, Delgado S, Castells A, Tasende M, Momblan D, Diaz del Gobbo G, et al. Transanal total mesorectal excision in rectal cancer: short-term outcomes in comparison with laparoscopic surgery. Annals of surgery. 2015; 261: 221-7.

23. Lelong B, Meillat H, Zemmour C, Poizat F, Ewald J, Mege D, et al. Short- and Mid-Term Outcomes after Endoscopic Transanal or Laparoscopic Transabdominal Total Mesorectal Excision for Low Rectal Cancer: A Single Institutional Case-Control Study. Journal of the American College of Surgeons. 2017; 224: 917-25

24. Perdawood SK, Al Khefagie GA. Transanal vs laparoscopic total mesorectal excision for rectal cancer: initial experience from Denmark. Colorectal disease : the official journal of the Association of Coloproctology of Great Britain and Ireland. 2016; 18: 51-8.

25. Perdawood SK, Thinggaard BS, Bjoern MX. Effect of transanal total mesorectal excision for rectal cancer: comparison of short-term outcomes with laparoscopic and open surgeries. Surgical endoscopy. 2017.

26. Rasulov AO, Mamedli ZZ, Gordeyev SS, Kozlov NA, Dzhumabaev HE. Short-term outcomes after transanal and laparoscopic total mesorectal excision for rectal cancer. Techniques in coloproctology. 2016; 20: 227-34.

27. Velthuis S, Nieuwenhuis DH, Ruijter TE, Cuesta MA, Bonjer HJ, Sietses C. Transanal versus traditional laparoscopic total mesorectal excision for rectal carcinoma. Surgical endoscopy. 2014; 28: 3494-9.

28. Quirke P, Durdey P, Dixon MF, Williams NS. Local recurrence of rectal adenocarcinoma due to inadequate surgical resection. Histopathological study of lateral tumour spread and surgical excision. Lancet (London, England). 1986; 2: 996-9.

29. Nagtegaal ID, van de Velde CJ, van der Worp E, Kapiteijn E, Quirke P, van Krieken JH. Macroscopic evaluation of rectal cancer resection specimen: clinical significance of the pathologist in quality control. Journal of clinical oncology : official journal of the American Society of Clinical Oncology. 2002; 20: $1729-34$.

30. Dindo D, Demartines N, Clavien PA. Classification of surgical complications: a new proposal with evaluation in a cohort of 6336 patients and results of a survey. Annals of surgery. 2004; 240: 205-13.

31. Jeong SY, Park JW, Nam BH, Kim S, Kang SB, Lim SB, et al. Open versus laparoscopic surgery for mid-rectal or low-rectal cancer after neoadjuvant chemoradiotherapy (COREAN trial): survival outcomes of an open-label, non-inferiority, randomised controlled trial. The Lancet Oncology. 2014; 15: 767-74.

32. Trastulli S, Cirocchi R, Listorti C, Cavaliere D, Avenia N, Gulla N, et al. Laparoscopic vs open resection for rectal cancer: a meta-analysis of randomized clinical trials. Colorectal disease : the official journal of the Association of Coloproctology of Great Britain and Ireland. 2012; 14: e277-96.

33. Fleshman J, Branda M, Sargent DJ, Boller AM, George V, Abbas M, et al. Effect of Laparoscopic-Assisted Resection vs Open Resection of Stage II or III Rectal Cancer on Pathologic Outcomes: The ACOSOG Z6051 Randomized Clinical Trial. Jama. 2015; 314: 1346-55.

34. Stevenson AR, Solomon MJ, Lumley JW, Hewett P, Clouston AD, Gebski VJ, et al. Effect of Laparoscopic-Assisted Resection vs Open Resection on Pathological Outcomes in Rectal Cancer: The ALaCaRT Randomized Clinical Trial. Jama. 2015; 314: 1356-63. 
35. Nagtegaal ID, Quirke P. What is the role for the circumferential margin in the modern treatment of rectal cancer? Journal of clinical oncology : official journal of the American Society of Clinical Oncology. 2008; 26: 303-12.

36. Nagtegaal ID, Marijnen CA, Kranenbarg EK, van de Velde CJ, van Krieken JH. Circumferential margin involvement is still an important predictor of local recurrence in rectal carcinoma: not one millimeter but two millimeters is the limit. The American journal of surgical pathology. 2002; 26: 350-7.

37. Marks JH, Myers EA, Zeger EL, Denittis AS, Gummadi M, Marks GJ. Long-term outcomes by a transanal approach to total mesorectal excision for rectal cancer. Surgical endoscopy. 2017; 31: 5248-57.

38. Thorpe H, Jayne DG, Guillou PJ, Quirke P, Copeland J, Brown JM. Patient factors influencing conversion from laparoscopically assisted to open surgery for colorectal cancer. The British journal of surgery. 2008; 95: 199-205.

39. Ito M, Sugito M, Kobayashi A, Nishizawa Y, Tsunoda Y, Saito N. Relationship between multiple numbers of stapler firings during rectal division and anastomotic leakage after laparoscopic rectal resection. International journal of colorectal disease. 2008; 23: 703-7.

40. Penna M, Hompes R, Arnold S, Wynn G, Austin R, Warusavitarne J, et al. Incidence and Risk Factors for Anastomotic Failure in 1594 Patients Treated by Transanal Total Mesorectal Excision: Results From the International TaTME Registry. Annals of surgery. 2018

41. Rouanet P, Mourregot A, Azar CC, Carrere S, Gutowski M, Quenet F, et al. Transanal endoscopic proctectomy: an innovative procedure for difficult resection of rectal tumors in men with narrow pelvis. Diseases of the colon and rectum. 2013; 56: 408-15.

42. Barendse RM, Oors JM, de Graaf EJ, Bemelman WA, Fockens P, Dekker E, et al. The effect of endoscopic mucosal resection and transanal endoscopic microsurgery on anorectal function. Colorectal disease : the official journal of the Association of Coloproctology of Great Britain and Ireland. 2013; 15: e534-41.

43. Chi P, Chen Z, Lu X. Transanal Total Mesorectal Excision: Can it Achieve the Standard of TME? Annals of surgery. 2017; 266: e87-e8.

44. Deijen CL, Velthuis S, Tsai A, Mavroveli S, de Lange-de Klerk ES, Sietses C, et al. COLOR III: a multicentre randomised clinical trial comparing transanal TME versus laparoscopic TME for mid and low rectal cancer. Surgical endoscopy. 2016; 30: 3210-5.

45. Lelong B, de Chaisemartin C, Meillat H, Cournier S, Boher JM, Genre D, et al. A multicentre randomised controlled trial to evaluate the efficacy, morbidity and functional outcome of endoscopic transanal proctectomy versus laparoscopic proctectomy for low-lying rectal cancer (ETAP-GRECCAR 11 TRIAL): rationale and design. BMC cancer. 2017; 17: 253.

46. Francis N, Penna M, Mackenzie $H$, et al. Consensus on structured training curriculum for transanal total mesorectal excision (TaTME). Surg Endosc. 2017; 31: 2711-9.

47. Maykel JA, Phatak UR, Suwanabol PA, Schlussel AT, Davids JS, Sturrock PR, et al. Initiation of a Transanal Total Mesorectal Excision Program at an Academic Training Program: Evaluating Patient Safety and Quality Outcomes. Diseases of the colon and rectum. 2017; 60: 1267-72. 